\title{
Laparoscopic anti-reflux surgery for idiopathic pulmonary fibrosis at a single centre
}

\author{
Ganesh Raghu1, Ellen Morrow ${ }^{2}$, Bridget F. Collins ${ }^{1}$, Lawrence A.T. Ho ${ }^{1}$, \\ Marcelo W. Hinojosa ${ }^{2}$, Jennifer M. Hayes ${ }^{1}$, Carolyn A. Spada ${ }^{1}$, \\ Brant Oelschlager ${ }^{2}$, Chenxiang Li ${ }^{3}$, Eric Yow ${ }^{3}$, Kevin J. Anstrom ${ }^{3}$, Dylan Mart ${ }^{2}$, \\ Keliang $\mathrm{Xiao}^{2}$ and Carlos A. Pellegrini ${ }^{2}$
}

\section{Affiliations:}

${ }^{1}$ Dept of Medicine, Center for Interstitial Lung Diseases, University of Washington Medical Center, Seattle, WA, USA.

${ }^{2}$ Dept of Surgery, University of Washington Medical Center, Seattle, WA, USA.

${ }^{3}$ Dept of Biostatistics and Bioinformatics, Duke University, Durham, NC, USA.

\section{Correspondence:}

Ganesh Raghu, Box 356175, Center for Interstitial Lung Diseases, UW Medicine, University of Washington, 1959 NE Pacific St, Seattle, WA 98195, USA.

E-mail: graghuduw.edu

ABSTRACT We sought to assess whether laparoscopic anti-reflux surgery (LARS) is associated with decreased rates of disease progression in patients with idiopathic pulmonary fibrosis (IPF).

The study was a retrospective single-centre study of IPF patients with worsening symptoms and pulmonary function despite antacid treatment for abnormal acid gastro-oesophageal reflux. The period of exposure to LARS was September 1998 to December 2012. The primary end-point was a longitudinal change in forced vital capacity (FVC) \% predicted in the pre- versus post-surgery periods.

27 patients with progressive IPF underwent LARS. At time of surgery, the mean age was 65 years and mean FVC was $71.7 \%$ pred. Using a regression model, the estimated benefit of surgery in FVC \% pred over 1 year was $5.7 \%$ (95\% CI $-0.9-12.2 \%, \mathrm{p}=0.088)$ with estimated benefit in FVC of $0.22 \mathrm{~L}$ (95\% CI $-0.06-0.49 \mathrm{~L}, \mathrm{p}=0.12)$. Mean DeMeester scores decreased from 42 to $4(\mathrm{p}<0.01)$. There were no deaths in the 90 days following surgery and $81.5 \%$ of participants were alive 2 years after surgery.

Patients with IPF tolerated the LARS well. There were no statistically significant differences in rates of FVC decline pre- and post-LARS over 1 year; a possible trend toward stabilisation in observed FVC warrants prospective studies. The ongoing prospective randomised controlled trial will hopefully provide further insights regarding the safety and potential efficacy of LARS in IPF.

@ERSpublications

Laparoscopic anti-reflux surgery is well tolerated in IPF, with a nonsignificant trend towards decreased FVC decline http://ow.ly/BspI300pbJh

Editorial comment in Eur Respir J 2016; 48: 623-625.

Received: March 072016 | Accepted after revision: May 162016 | First published online: Aug 042016

Conflict of interest: Disclosures can be found alongside this article at erj.ersjournals.com

Copyright OERS 2016 


\section{Introduction}

Idiopathic pulmonary fibrosis (IPF) is a progressive fibrotic lung disease that usually leads to death within 5 years of diagnosis $[1,2]$. While two drugs, pirfenidone and nintedanib, have recently been shown to reduce disease progression as measured by changes in forced vital capacity (FVC) over 52 weeks, additional treatment strategies to abort disease progression and enhance survival are needed [3, 4].

Prevention of recurrent insults to the lung epithelium may play a role in preserving lung function [5]. There is increasing evidence to support the concept of abnormal gastro-oesophageal reflux (GOR) and micro-aspiration in the pathogenesis of IPF $[6,7]$. Antacid treatment with proton pump inhibitors (PPIs) and $\mathrm{H} 2$ receptor antagonists is known to suppress acidity of gastric contents, but does not suppress GOR. Other components of gastric effluent in refluxate, including pepsin, may still reach the lung by mircroaspiration and cause acute exacerbation of IPF [6-8]. Surgical correction of abnormal GOR may be an appropriate treatment for patients with IPF to minimise further insults to the lung by refluxate. We hypothesised that laparoscopic anti-reflux surgery (LARS) would result in a stabilisation or slower decline of FVC compared to pre-LARS rates of decline among patients with IPF by "preventing" further insults to the lung by GOR.

\section{Methods \\ Study population}

This was a retrospective cohort study of patients with IPF who were managed and subjected to LARS at University of Washington Medical Center (UWMC; Seattle, WA, USA) between September 1998 and December 2012. Inclusion criteria were IPF patients meeting 2011-published criteria for diagnosis of IPF who had worsening shortness of breath or cough and $\geqslant 5 \%$ decrease in FVC during the preceding year despite antacid treatment and adoption of lifestyle modifications to reduce GOR, diagnosis of abnormal acid GOR by $24 \mathrm{~h} \mathrm{pH}$ study with DeMeester score $>14.7$ and post-operative pulmonary function tests (PFTs) measured without an intervening lung transplant. All clinical data were assessed to ensure that included patients met diagnostic criteria per the 2011 IPF guidelines; patients who entered the study before publication of the 2011 guidelines were subsequently discussed in multidisciplinary meeting to ensure that they met 2011 guideline criteria for IPF, and indeed, all did meet the criteria [1]. Exclusion criteria were patients participating in clinical trials; severe pulmonary hypertension (defined as mean pulmonary artery pressure $\geqslant 50 \mathrm{mmHg}$ by right heart catheterisation), morbid obesity and other contraindications to general anaesthesia.

\section{Patient flow and disposition}

All patients who met study criteria were evaluated for consideration for LARS at the Center for Esophageal and Gastric Surgery at the University of Washington. 41 patients with IPF who had abnormal GOR underwent LARS during the study time period; 14 of these patients underwent surgery as a requirement to be listed for lung transplantation (in keeping with a policy at our programme to minimise risk of lung allograft airway complications). Since lung transplant occurred prior to obtaining PFTs post-LARS, these patients were excluded. Therefore, 27 patients with IPF who underwent LARS and underwent PFTs during the year following LARS were included. Perioperative care and LARS were performed as per standard of care for patients undergoing surgery at our centre.

\section{Data collection}

Patients were followed based on usual standard of care to monitor disease course. Data gathered for clinically relevant purposes were analysed retrospectively in accordance with approval by the UWMC human subjects review board.

\section{End-points}

Efficacy end-points were based on longitudinal assessments of FVC (L and \% predicted), diffusing capacity of the lung for carbon monoxide (DLCO) and DLCO \% pred obtained pre- and post-LARS. Safety end-points were perioperative mortality (death within 90 days of LARS) or morbidity with particular attention to respiratory events. Acute respiratory decline was defined as worsened dyspnoea and oxygenation requiring more supplemental oxygen than baseline within 90 days of LARS.

\section{Statistical analysis}

Baseline characteristics are presented using mean (minimum-maximum) for continuous variables and counts with percentages for categorical variables. Baseline characteristics were determined at the visit immediately preceding LARS. PFTs and $\mathrm{pH}$ measurements within each patient were compared between the first assessment post-LARS and the last assessment pre-LARS using a paired t-test. 
The primary analysis was based on a comparison of changes in the trajectory between FVC \% pred measurements pre-LARS and FVC \% pred measurements post-LARS. Only spirometry results within 2 years of LARS were included in data analysis. A mixed-effect model was built to estimate the change in lung function 1 year after surgery compared to standard of care (without LARS). For each patient, there were up to two measurements prior to surgery and up to three measurements after surgery. In the primary analysis, a mixed model was used with FVC \% pred as the outcome, and the predictor variables included separate intercept and slope terms for the pre- and post-LARS periods. Time was modelled as the days prior to or following LARS. Repeated measurements were modelled with a combination of compound symmetry and autoregressive covariance structure. This model assumes that the correlation between measurements decreases over time. An estimate statement was used to estimate the expected benefit of LARS at 12 months following surgery.

Long-term survival was assessed using a Kaplan-Meier analysis. We censored data from all patients not known to be deceased as of September 1, 2015. p-values $<0.05$ were considered statistically significant. All data analyses were performed using SAS 9.3 (Cary, NC, USA) or R software (version 3.0.3; www.r-project.org).

\section{Results}

\section{Cohort and baseline demographics}

Mean age was 65 years, 55.6\% were male and most were former smokers (table 1). Mean FVC and DLCO (corrected to haemoglobin) were $71.7 \%$ pred and $43.7 \%$ pred, respectively. Mean \pm SD time interval between IPF diagnosis and LARS was $1.7 \pm 1.6$ years, excluding one outlier value of 13.3 years (the next greatest value was 5.5 years).

\section{Pattern on high-resolution computed tomography}

On high-resolution computed tomography, 15 (55.6\%) patients had definite usual interstitial pneumonia (UIP), eight (29.6\%) had possible UIP and four (14.8\%) had a pattern inconsistent with UIP. Some of the patients with UIP had a surgical lung biopsy prior to presentation to our centre. Patients with patterns of possible UIP and inconsistent UIP were accepted to have IPF in the appropriate clinical setting if the surgical lung biopsy had features of UIP pattern.

\section{Assessment of GOR}

All patients were subjected to $24 \mathrm{~h} \mathrm{pH}$ monitoring and oesophageal manometry pre-operatively. Three of the 27 patients who underwent the preoperative $24 \mathrm{~h} \mathrm{pH}$ monitoring could not tolerate being off antacid treatment during the 7 days preceding $24 \mathrm{~h} \mathrm{pH}$ monitoring (required for acid GOR assessment) due to

$\begin{array}{lc}\text { TABLE } 1 \text { Baseline demographics } & \\ \text { Subjects } & 27 \\ \text { Age at surgery years } & 65(51-77) \\ \text { Male } & 15(55.6) \\ \text { BMI kg.m }{ }^{-2} & 28.5(21.6-36) \\ \text { Smoking status \% } & \\ \quad \text { Never-smoker } & 11(40.7) \\ \text { Former smoker } & 16(59.3) \\ \text { Current smoker } & 0(0) \\ \text { Lung physiology features } & \\ \text { FVC \% pred } & 71.7(45-116) \\ \text { FEV } 1 / \text { FVC } & 0.78(0.58-0.88) \\ \text { DLCo \% pred } & 43.7(22-70) \\ \text { Supplemental oxygen usage } & 13(48.1) \\ \text { Initial walk distance m } & 385.5(79.2-562) \\ \text { Surgical lung biopsy } & 15(55.6) \\ \text { IPF diagnosis to LARS years } & 1.7 \pm 1.6 \\ \text { Pulmonary artery pressures mmHg } & \\ \quad \text { Pulmonary artery systolic pressure: echocardiogram } & 34.2(23-45) \\ \text { Mean pulmonary artery pressure: right heart catheterisation } & 21.8(8-42) \\ \text { DeMeester composite score } & 36.2(1-112.7)\end{array}$

Data are presented as $\mathrm{n}$, mean (minimum-maximum), $\mathrm{n}(\%)$ or mean \pm SD. BMI: body mass index; FVC: forced vital capacity; FEV1: forced expiratory volume in $1 \mathrm{~s}$; DLCO: diffusion capacity of the lung for carbon monoxide; IPF: idiopathic pulmonary fibrosis; LARS: laparoscopic anti-reflux surgery. \#: corrected to haemoglobin; ${ }^{\text {: }}$ : for 26 patients, outlier value (13.3 years) excluded. 
severe GOR disease symptoms, and $\mathrm{pH}$ monitoring was performed while they were on PPI treatment; these three patients had normal DeMeester scores, but had evidence of GOR by barium oesophagogram and/or impedance. The other 24 patients had abnormal DeMeester scores $>14.7$, consistent with abnormal acid GOR.

\section{Assessment of pulmonary function}

Individual patient-level measurements of FVC ( $\mathrm{L}$ and \% pred) are shown in figure 1. The mean time between the last pre-surgery and first post-surgery FVC assessments was 185 days. Over that period of time the mean \pm SD change in FVC ( $\mathrm{L}$ and \% pred) were $0.05 \pm 0.25 \mathrm{~L}(\mathrm{p}=0.32)$ and $1.5 \pm 5.6 \%(\mathrm{p}=0.15)$, respectively.

Using a regression model, the estimated benefit of surgery in FVC at 1 year post-surgery was $5.7 \%$ pred (95\% CI $-0.9-12.2 \%, \mathrm{p}=0.088)$ and $0.22 \mathrm{~L}(95 \% \mathrm{CI}-0.06-0.49 \mathrm{~L}, \mathrm{p}=0.12)$. In both cases comparison is based on the regression estimate of the pre-surgery slope (figure 1).

\section{Perioperative safety}

Average post-operative hospital stay was 2 days (range 1-6 days). There were no intra-operative complications. Within 30 days following LARS four patients had complications: two had dysphagia requiring readmission and endoscopic dilation; one had nausea requiring readmission; and one had urinary retention. There were no deaths or acute exacerbations of IPF within the 90 days following LARS. Two re-operations were noted during the follow-up period: one patient was converted to a Dor, a form of partial fundoplication, 3 months post-operatively; and one repeat LARS was performed 12 years after the initial LARS for recurrence of GOR and further deterioration in pulmonary function.

\section{Reassessment of $\mathrm{pH}$ following LARS}

At $\sim 6$ months post-LARS, 20 out of 27 patients underwent repeat pH probe testing. In 19 of these 20 patients, oesophageal $\mathrm{pH}$ had normalised with the mean DeMeester score decreasing from 42 to 4 $(\mathrm{p}<0.01)$.

\section{Long-term survival}

Among the 27 patients in the cohort, 11 died during follow-up. There were no deaths in the 90 days following LARS and $81.5 \%$ of participants were alive at 2 years following LARS (figure 2).

\section{Discussion}

The exact pathogenesis of IPF is unclear, but probably involves recurrent injury to alveolar epithelial cells (AEC) in genetically susceptible patients $[5,9,10]$. Sources of potential injury include chronic microaspiration of gastro-oesphageal refluxate, cigarette smoking and viral infections [5, 11-14]. While much investigation has been centred on causes of AEC injury in IPF, our study is the first to assess a strategy to prevent further injury by surgical intervention. We hypothesised that microaspiration of both acidic and alkaline GOR plays a role in AEC injury and that surgical intervention to reduce or halt this process would slow the rate of decline in pulmonary function seen in patients with IPF. Our results suggest a trend toward decreased rates of decline in FVC following LARS, but did not reach statistical significance, and therefore are inconclusive. While patients with IPF are generally expected to be at higher perioperative risk for surgery, in our experience at a single centre LARS was safe in these patients, with no major adverse events observed.
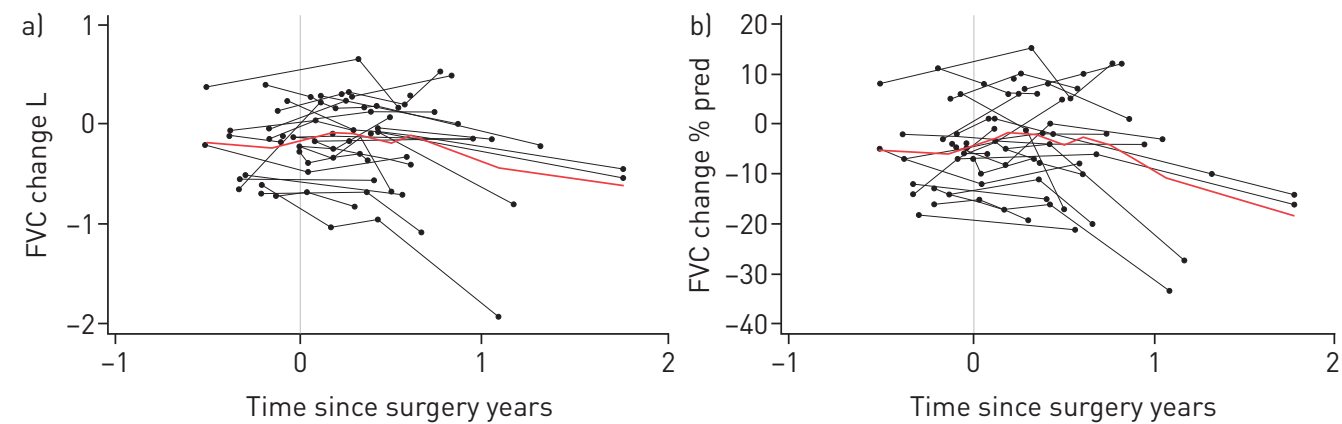

FIGURE 1 Change in forced vital capacity (FVC) a) volume and b) \% predicted over time pre- and postlaparoscopic anti-reflux surgery for 27 patients with idiopathic pulmonary fibrosis. Locally weighted scatterplot smoothing regression curves are shown in in red. 


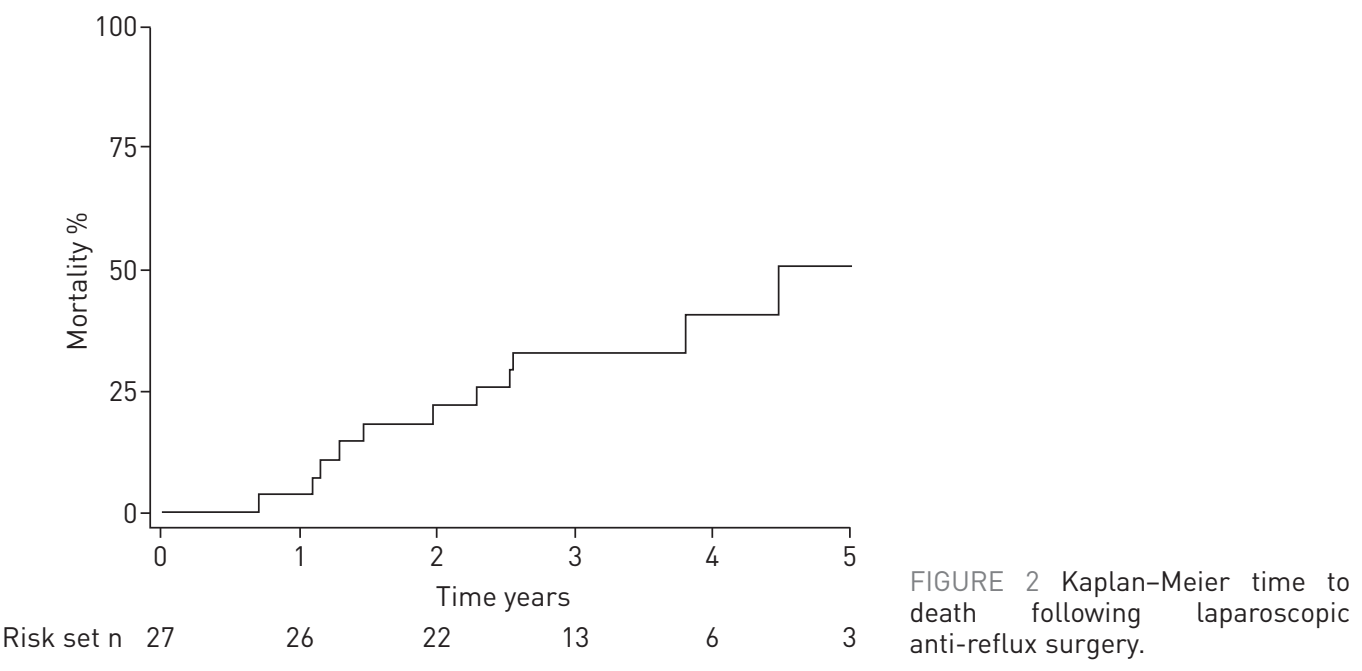

Up to $90 \%$ of patients with IPF have abnormal acid GOR, which is often clinically silent $[15,16]$. Analysis of data from three randomised controlled trials of IPF patients demonstrated that antacid therapy was associated with a reduced rate of decline in pulmonary function and fewer acute exacerbations of IPF [17]. Stabilisation of pulmonary function with treatment of GOR and longer survival among IPF patients treated with antacid medications or Nissen fundoplication has been observed in case series and retrospective studies [18-20]. Acknowledging the low quality of evidence from these and other studies, the 2011 and 2015 guidelines for treatment of IPF made a conditional recommendation for antacid treatment in all IPF patients $[1,2]$. Given that nonacidic components of gastric juice (e.g. pepsin) have been found in bronchoalveolar lavage fluid of patients with IPF and acute exacerbation of IPF, it is possible that controlling the presence and volume of any refluxate reaching the alveoli may be preferable to merely controlling acidity $[7,21]$.

Among patients with IPF in recent phase III clinical trials, the decline in FVC per year has varied from $190 \mathrm{~mL}$ over 60 weeks (PANTHER) to $240 \mathrm{~mL}$ per year (INPULSIS) to $280 \mathrm{~mL}$ per year (ASCEND) in patients randomised to the placebo arms $[3,4,22]$. The estimated rate of decline in FVC prior to LARS in our cohort of patients with IPF was $319 \mathrm{~mL}$ per year, worse than that in patients enrolled in recent clinical trials. By comparison, the estimated decline in FVC over the first year following LARS was $102 \mathrm{~mL}$, a relatively lesser (but not statistically significant) decline than the observed declines of $114.7 \mathrm{~mL}$ and $235 \mathrm{~mL}$ over 1 year of nintedanib and pirfenidone therapy, respectively. Since the rate of decline in FVC among patients with IPF over the disease course is not linear, our results may simply a represent natural variation in disease course $[3,4]$. While the observed trend warrants further prospective studies in well-designed studies with controls, we cannot conclude from our study that LARS is effective treatment for IPF.

Patients in our study were, on average, relatively young (mean age 65 years), although this is similar to the mean age in recent randomised controlled trials of patients with $\operatorname{IPF}[3,4,23]$. Mean FVC was relatively preserved, which is again similar to that seen in recent clinical trials [3, 4, 23]. Similar to findings of these clinical trials, our results may only be applicable to IPF patients who are similar in age, demographics and baseline pulmonary function to those in our study, not to all IPF patients. While median survival from time of diagnosis in IPF is $2-3$ years, $81.5 \%$ of the patients in our cohort were alive at 2 years following LARS [1]. Mean time from IPF diagnosis to LARS among patients in our study was 1.7 years. Therefore, after two additional years of follow-up, our expectation would be that $<50 \%$ of our cohort would have survived. However, from our findings we cannot determine whether or to what extent LARS may have contributed to this survival rate.

Patients with IPF, who are often older and may have comorbid pulmonary hypertension, are at increased risk of peri- and post-operative complications $[24,25]$. While patients with severe pulmonary hypertension were not subjected to LARS in our study, we did not observe any intra-operative complications, deaths or acute exacerbations of IPF among patients in our cohort in the 90 days following LARS. This may have been due in part to our small sample size. Post-operative complications were minimal, and lead to re-operation in two patients. While LARS was well tolerated among patients with IPF in our study, some of whom had severe disease, we emphasise that our observations are from a single centre experienced in management of IPF and LARS. Results of prospective studies in multiple centres are needed to determine the safety and efficacy of LARS in similar patients with IPF. 
Our study has limitations, including those inherent to retrospective studies without controls. Although our centre is known to evaluate and manage a large number of patients with IPF, the small number of subjects in this study can be explained by several factors: 1) selection bias in our sample based on strict inclusion and exclusion criteria; 2) all patients were required to have a decline in pulmonary function prior to receiving LARS (any benefit may have been the result of regression to the mean); 3) LARS is not an approved treatment for IPF, so was performed only in patients willing to undergo LARS based on documented abnormal GOR and progressive IPF despite treatment with antacids; and 4) the study was designed specifically to assess disease progression following LARS and to attribute findings to LARS alone, we eliminated all other patients with confounding factors including concomitant treatment regimens/ participation in clinical trials that could potentially modulate disease progression and changes in PFTs [26]. Additionally, PFTs were not collected at set time intervals, as would be the case in a prospective study. That acknowledged, there were no other changes in treatment regimen among patients in our study population over the time period of interest. None of the patients in our cohort were participating in the PANTHER study or in any other clinical trials; none were taking prednisone $>10 \mathrm{mg}^{-d_{a y}{ }^{-1}}$ or azathioprine. Since at the time of the study, patients were aware of the potential beneficial effects of $\mathrm{N}$-acetylcysteine (NAC), we allowed them to take over-the-counter NAC as a supplement, although NAC has since not been shown to universally affect FVC in IPF [23]. Despite the limitations, some aspects of our study design may be regarded as relative strengths allowing for consistency in procedures. Regardless, results from our small sample raise the possibility that LARS could be a treatment strategy for patients with IPF and abnormal acid GOR. This needs to be investigated further in prospective studies.

\section{Conclusion}

We describe a novel treatment strategy to prevent further injury to the fibrotic lung in well-defined patients with progressive IPF and GORD. Treatment with LARS resulted in normalisation of acid exposure in the distal oesophagus and was safe and well tolerated in our patient population. We observed a non-significant trend toward a slower rate of decline in pulmonary function over 1 year post-LARS compared to the pre-LARS period than that typically seen in patients with progressive IPF, which warrants further prospective study. It is hoped that the ongoing phase 2 multicentre clinical trial WRAP-IPF (NCT 01982968) will provide additional, necessary insights regarding the efficacy and safety of LARS and enhance understanding of the management of GOR among patients with IPF.

\section{References}

1 Raghu G, Collard HR, Egan JJ. An official ATS/ERS/JRS/ALAT statement: idiopathic pulmonary fibrosis: evidence-based guidelines for diagnosis and management. Am J Respir Crit Care Med 2011; 183: 788-824.

2 Raghu G, Rochwerg B, Zhang Y, et al. An official ATS/ERS/JRS/ALAT clinical practice guideline: treatment of idiopathic pulmonary fibrosis. An update of the 2011 clinical practice guideline. Am J Respir Crit Care Med 2015; 192: e3-e19.

3 Richeldi L, du Bois RM, Raghu G, et al. Efficacy and safety of nintedanib in idiopathic pulmonary fibrosis. $N$ Engl J Med 2014; 370: 2071-2082.

4 King TE Jr, Bradford WZ, Fagan EA, et al. A phase 3 trial of pirfenidone in patients with idiopathic pulmonary fibrosis. N Engl J Med 2014; 370: 2083-2092.

$5 \quad$ King TE Jr, Pardo A, Selman M. Idiopathic pulmonary fibrosis. Lancet 2011; 378: 1949-1961.

6 Raghu G, Meyer KC. Silent gastro-oesophageal reflux and microaspiration in IPF: mounting evidence for anti-reflux therapy? Eur Respir J 2012; 39: 242-245.

7 Lee JS, Song JW, Wolters PJ, et al. Bronchoalveolar lavage pepsin in acute exacerbation of idiopathic pulmonary fibrosis. Eur Respir J 2012; 39: 352-358.

8 Raghu G. Idiopathic pulmonary fibrosis: increased survival with "gastroesophageal reflux therapy": fact or fallacy? Am J Respir Crit Care Med 2011; 184: 1330-1332.

9 Fingerlin TE, Murphy E, Zhang W, et al. Genome-wide association study identifies multiple susceptibility loci for pulmonary fibrosis. Nat Genet 2013; 45: 613-620.

10 Ahluwalia N, Shea BS, Tager AM. New therapeutic targets in idiopathic pulmonary fibrosis. Aiming to rein in runaway wound-healing responses. Am J Respir Crit Care Med 2014; 190: 867-878.

11 Lee JS, Collard HR, Raghu G, et al. Does chronic microaspiration cause idiopathic pulmonary fibrosis? Am J Med 2010; 123: 304-311.

12 Tang YW, Johnson JE, Browning PJ, et al. Herpesvirus DNA is consistently detected in lungs of patients with idiopathic pulmonary fibrosis. J Clin Microbiol 2003; 41: 2633-2640.

13 Tanjore H, Blackwell TS, Lawson WE. Emerging evidence for endoplasmic reticulum stress in the pathogenesis of idiopathic pulmonary fibrosis. Am J Physiol Lung Cell Mol Physiol 2012; 302: L721-L729.

14 Kelly BG, Lok SS, Hasleton PS, et al. A rearranged form of Epstein-Barr virus DNA is associated with idiopathic pulmonary fibrosis. Am J Respir Crit Care Med 2002; 166: 510-513.

15 Tobin RW, Pope CE, Pellegrini CA, et al. Increased prevalence of gastroesophageal reflux in patients with idiopathic pulmonary fibrosis. Am J Respir Crit Care Med 1998; 158: 1804-1808.

16 Raghu G, Freudenberger TD, Yang S, et al. High prevalence of abnormal acid gastro-oesophageal reflux in idiopathic pulmonary fibrosis. Eur Respir J 2006; 27: 136-142.

17 Lee JS, Collard HR, Anstrom KJ, et al. Anti-acid treatment and disease progression in idiopathic pulmonary fibrosis: an analysis of data from three randomized controlled trials. Lancet Respir Med 2013; 1: 369-376. 
18 Raghu G, Yang ST, Spada C, et al. Sole treatment of acid gastroesophageal reflux in idiopathic pulmonary fibrosis: a case series. Chest 2006; 129: 794-800.

19 Linden PA, Gilbert RJ, Yeap BY, et al. Laparoscopic fundoplication in patients with end-stage lung disease awaiting transplantation. J Thorac Cardiovasc Surg 2006; 131: 438-446.

20 Lee JS, Ryu JH, Elicker BM, et al. Gastroesphageal reflux therapy is associated with longer survival in patients with idiopathic pulmonary fibrosis. Am J Respir Crit Care Med 2011; 184: 1390-1394.

21 Fisichella PM, Davis CS, Lundberg PW, et al. The protective role of laparoscopic antireflux surgery against aspiration of pepsin after lung transplantation. Surgery 2011; 150: 598-606.

22 Idiopathic Pulmonary Fibrosis Clinical Research Network, Raghu G, Anstrom KJ, et al. Prednisone, azathioprine, and $N$-acetylcysteine for pulmonary fibrosis. N Engl J Med 2012; 366: 1968-1977.

23 Idiopathic Pulmonary Fibrosis Clinical Research Network, Martinez FJ, de Andrade JA, et al. Randomized trial of acetylcysteine in idiopathic pulmonary fibrosis. N Engl J Med 2014; 370: 2093-2101.

24 Smetana GW, Lawrence VA, Cornell JE. Preoperative pulmonary risk stratification for noncardiothoracic surgery: systematic review for the American College of Physicians. Ann Intern Med 2006; 144: 581-595.

25 Fleisher LA, Beckman JA, Brown KA, et al. ACC/AHA 2007 guidelines on perioperative cardiovascular evaluation and care for noncardiac surgery. Circulation 2007; 116: e418-e499.

26 Bland JM, Altman DG. Regression towards the mean. BMJ 1994; 308: 1499. 